\section{International Scientific Journal}

\section{Theoretical \& Applied Science}

p-ISSN: 2308-4944 (print) ｅ-ISSN: 2409-0085 (online)

Year: $2015 \quad$ Issue: $09 \quad$ Volume: 29

Published: $30.09 .2015 \quad$ http://T-Science.org
Anna Sergeevna Kravchenko

$\mathrm{PhD}$ student of Department «Economic analysis»

Kuban State Agrarian University,

Krasnodar, Russia

anna.kravchenko.92@inbox.ru

SECTION 31. Economic research, finance,

innovation, risk management.

\title{
DEFINITION DIRECTIONS TO IMPROVE THE EFFICIENCY OF BUSINESS ACTIVITIES OF ORGANIZATIONS BASED ON THE ANALYSIS OF BALANCE THEIR DEVELOPMENT
}

\begin{abstract}
Defined the main factors affecting the efficiency of business activities. Explained the nature of their influence and the necessity of their integration. The analysis of balance development of particular company. Based on analysis proposed specific actions to improve the efficiency of its activities.

Key words: analysis, economic efficiency, balance development, resources, capital productivity, labor productivity, capital structure, asset turnover, return on sales.

Language: Russian English

Citation: Kravchenko AS (2015) DEFINITION DIRECTIONS TO IMPROVE THE EFFICIENCY OF BUSINESS ACTIVITIES OF ORGANIZATIONS BASED ON THE ANALYSIS OF BALANCE THEIR DEVELOPMENT. ISJ Theoretical \& Applied Science 09 (29): 131-136.

Soi: http://s-o-i.org/1.1/TAS-09-29-26 Doi: crossef http://dx.doi.org/10.15863/TAS.2015.09.29.26

\section{ОПРЕДЕЛЕНИЕ НАПРАВЛЕНИЙ ПОВЫШЕНИЯ ЭФФЕКТИВНОСТИ ПРЕДПРИНИМАТЕЛЬСКОЙ ДЕЯТЕЛЬНОСТИ ОРГАНИЗАЦИЙ НА ОСНОВЕ АНАЛИЗА СБАЛАНСИРОВАННОСТИ ИХ РАЗВИТИЯ}

Аннотация: Определены основные факторы, влияющчие на эффективность предпринимательской деятельности. Объяснена сущңность их влияния и обоснована необходимость их комплексного учета. Проведен анализ сбалансированности развития конкретного предприятия. По результатам анализа предложены конкретные мероприятия по повышению эффективности его деятельности.

Ключевые слова: анализ, экономическая эффективность, сбалансированность развития, ресурсы, фондоотдача, производительность труда, структура капитала, оборачиваемость активов, рентабельность продаж.
\end{abstract}

Эффективность деятельности организации комплексная экономическая категория, включающая в себя не только рациональное использование ресурсного потенциала, но и повышение качества выпускаемой продукции, степень удовлетворенности клиентов этой продукцией, которые находят свое выражение в скорости и доходности коммерческих операций, а также оптимальная структура имущества и источников его образования [8].

Только сбалансированность всех факторов эффективности деятельности позволит предприятию успешно функционировать и развиваться в условиях расширенного воспроизводства.

По нашему мнению на эффективность предпринимательской деятельности влияют

ISPC Modern research and development,

Philadelphia, USA следующие основные факторы:

1) обеспеченность и эффективность использования технических ресурсов, под которыми понимаются, прежде всего, основные средства.

Недостаточная обеспеченность техникой приводит к увеличению нагрузки на каждую ее единицу, что заставляет хозяйствующие субъекты более рационально подходить к вопросам организации труда и планирования технологического процесса, чтобы уменьшить простои оборудования. Вместе с тем, недостаток основных средств приводит к увеличению затрат времени и труда на совершение технологических процессов, а их избыток ведет к увеличению себестоимости производства, вследствие роста затрат на содержание и обслуживание 
неиспользуемой техники [2]. Поэтому оптимальная обеспеченность основными средствами выступает в качестве одного из ключевых факторов повышения эффективности деятельности.

От эффективности использования основных средств зависит эффективность использования трудовых ресурсов. Замена ручного труда механизированным и автоматизация производственных процессов способствуют увеличению эффективности использования рабочего времени и сокращению его потерь, что находит свое выражение в росте производительности труда работников.

Кроме того, увеличение фондоотдачи и производительности труда способствуют относительной экономии затрат и сопровождаются ростом рентабельности продаж, что свидетельствует о повышении эффективности коммерческих операций хозяйствующих субъектов;

2) обеспеченность и эффективность использования материальных ресурсов (прежде всего, материально-производственных запасов).

В отношении обеспеченности предприятий материальными ресурсами наблюдается та же тенденция, что и с техническими ресурсами. Чем в большей степени хозяйствующие субъекты обеспечены запасами, тем менее эффективно они их используют. Вместе с тем недостаток или нарушение ритмичности поставок сырья и материалов приводят к сбоям производственного процесса, вызывают простои оборудования и потери рабочего времени, что ведет к росту себестоимости производства и снижению конкурентоспособности продукции.

Увеличение эффективности использования материальных ресурсов напрямую не влияет на эффективность использования основных средств. Это объясняется тем, что с одной стороны в состав материальных ресурсов входят топливо и запасные части, расходы на которые являются существенной статьей материальных затрат и увеличиваются при покупке более мощной и современной техники. С другой стороны, использование такой техники приводит к относительной экономии затрат на производство продукции.

Повышение эффективности использования сырья и материалов происходит вследствие лучшей организации производства и совершенствования технологических процессов, что, в свою очередь, приводит к росту производительности труда [1].

Также по мере роста эффективности использования материальных ресурсов наблюдается относительная экономия материальных затрат, являющихся существенной частью себестоимости продукции любого предприятия. А это, в свою очередь, способствует росту рентабельности продаж;

3) обеспеченность и эффективность использования трудовых ресурсов.

Ограниченность трудовых ресурсов заставляет хозяйствующие субъекты уделять больше внимания вопросам организации труда, сокращения простоев и прогулов. Кроме того отмечается увеличение механизации и автоматизации производственных процессов, что в свою очередь приводит к росту не только производительности труда, но и фондоотдачи [2].

Таким образом, между производительностью труда и фондоотдачей наблюдается прямая зависимость: рост эффективности использования основных фондов сопровождается увеличением эффективности использования трудовых ресурсов. Это объясняется тесной связью между механизированным и ручным трудом.

Увеличение производительности труда также сопровождается ростом эффективности использования сырья и материалов вследствие лучшей организации производства и совершенствования технологических процессов.

Кроме того, рост производительности труда, сопровождающийся увеличением эффективности использования основных средств и материальнопроизводственных запасов, приводит к относительной экономии издержек производства, что, в свою очередь, способствует росту прибыли более быстрыми темпами, чем рост выручки [9].

Таким образом, обеспеченность и эффективность использования рассмотренных ресурсов находятся в тесной взаимосвязи и взаимозависимости. Изменение одной составляющей неизменно приводит к изменению другой, что обусловливает необходимость комплексного рассмотрения этих факторов;

4) коммерческая составляющая, выражающаяся в успешности организации на рынках сбыта, определяемой через скорость и доходность коммерческих операций. В наиболее общем виде она характеризуется коэффициентом оборачиваемости оборотных активов и рентабельностью продаж.

Повышение скорости оборота оборотных средств находит отражение в росте выручки, валового дохода и прибыли в расчете на единицу функционировавших в отчетном периоде оборотных средств. Вместе с тем экономное расходование материальных оборотных средств по своему эффекту равнозначно увеличению объема производства товаров без дополнительных затрат.

Коммерческая составляющая тесно связана с ранее рассмотренными факторами. Как уже отмечалось, увеличение фондоотдачи, материалоотдачи и производительности труда 
приводит к росту рентабельности продаж. Это объясняется тем, что для повышения эффективности использования основных средств, материально-производственных запасов и трудовых ресурсов требуется лучшая организация производственных и технологических процессов, что ведет к снижению себестоимости производства и увеличению прибыли. Кроме того, проведенное исследование показало, что при низких значениях производительности труда и фондоотдачи предприятия получают убыток от основной деятельности, вследствие существенного роста себестоимости производства, вызванного низкой эффективностью использования ресурсов.

Вместе с тем, для повышения эффективности деятельности недостаточно только рационального использования ресурсного потенциала. Эффективность проявляется в отношении полученного результата к произведенным затратам. А результат зависит не только от производственных факторов, но и ценовой политики, маркетинговой деятельности, удовлетворенности клиентов выпускаемой продукцией и т.д. Он находит свое выражение в объемах продаж и скорости коммерческих операций. Коммерческая составляющая не менее важна, чем производственная;

5) структура имущества и источников его образования.

Размещение средств предприятия имеет очень большое значение в финансовой деятельности и повышении ее эффективности. От того, какие средства вложены в основные и оборотные средства, сколько их находится в сфере производства и в сфере обращения, в денежной и материальной форме, насколько оптимально их соотношение во многом зависят результаты производственной и финансовой деятельности, а следовательно, и эффективность предпринимательской деятельности в целом. В связи с этим необходимо определить: какая структура имущества и источников его образования способствует увеличению эффективности деятельности [5].

По нашему мнению, в структуре активов должно наблюдаться оптимальное соотношение между основными средствами и запасами. С одной стороны при недостаточной обеспеченности техническими ресурсами накопление материально-производственных запасов представляется нецелесообразным вследствие отсутствия технической возможности их переработки. С другой стороны, высокий уровень оснащенности техническим ресурсами при отсутствии необходимых для обработки сырья и материалов приведет к простоям оборудования, удорожанию себестоимости производства и также является нерациональным. Кроме того, для обеспечения своевременных и бесперебойных поставок техники и материальнопроизводственных запасов у предприятия должен быть определенный объем денежной наличности.

В структуре пассивов должны преобладать наиболее дешевые источники финансирования, срок погашения которых должен соответствовать сроку использования активов, приобретаемых за их счет. Так, на приобретение основных средств и иных внеоборотных активов представляется целесообразным направлять собственные и долгосрочные заемные средства, а финансирование оборотных активов (в частности запасов) осуществлять за счет краткосрочных источников. С одной стороны это способствует поддержанию достаточного уровня ликвидности и платежеспособности, а с другой стороны - не приведет к появлению излишних финансовых расходов по обслуживанию заемных средств.

Комплексное рассмотрение всех перечисленных факторов позволит выявить сбалансированность развития хозяйствующих субъектов или наоборот, выявит его «однобокость».

Рассмотрим применение комплексного подхода к анализу и оценке эффективности предпринимательской деятельности на примере ФГУП им. Калинина Павловского района Краснодарского края.

В качестве базы сравнения мы использовали среднеотраслевые показатели деятельности, определенные по данным 170 средних и крупных сельскохозяйственных организаций Краснодарского края. Используя линейное выравнивание среднеотраслевых значений по каждому из вышерассмотренных факторов, мы определили «нормальные» для ФГУП им. Калинина при сложившейся структуре имущества и источников его образования, положения на рынках сбыта, а также текущего уровня обеспеченности техническими, материальными и трудовыми ресурсами показатели эффективности деятельности. Фактические и нормальные значения рассматриваемых показателей представлены на рисунке 1. 


\begin{tabular}{|c|c|c|c|c|c|c|}
\hline Impact Factor: & $\begin{array}{l}\text { ISRA (India) } \\
\text { ISI (Dubai, UAE } \\
\text { GIF (Australia) } \\
\text { JIF }\end{array}$ & $\begin{array}{l}=1.344 \\
=0.829 \\
=0.356 \\
=1.500\end{array}$ & $\begin{array}{l}\text { SIS (USA) } \\
\text { PИHЦ (Russia) } \\
\text { ESJI (KZ) } \\
\text { SJIF (Morocco) }\end{array}$ & $\begin{array}{l}=0.912 \\
=0.179 \\
=1.042 \\
=2.031\end{array}$ & ICV (Poland) & $=6.630$ \\
\hline
\end{tabular}

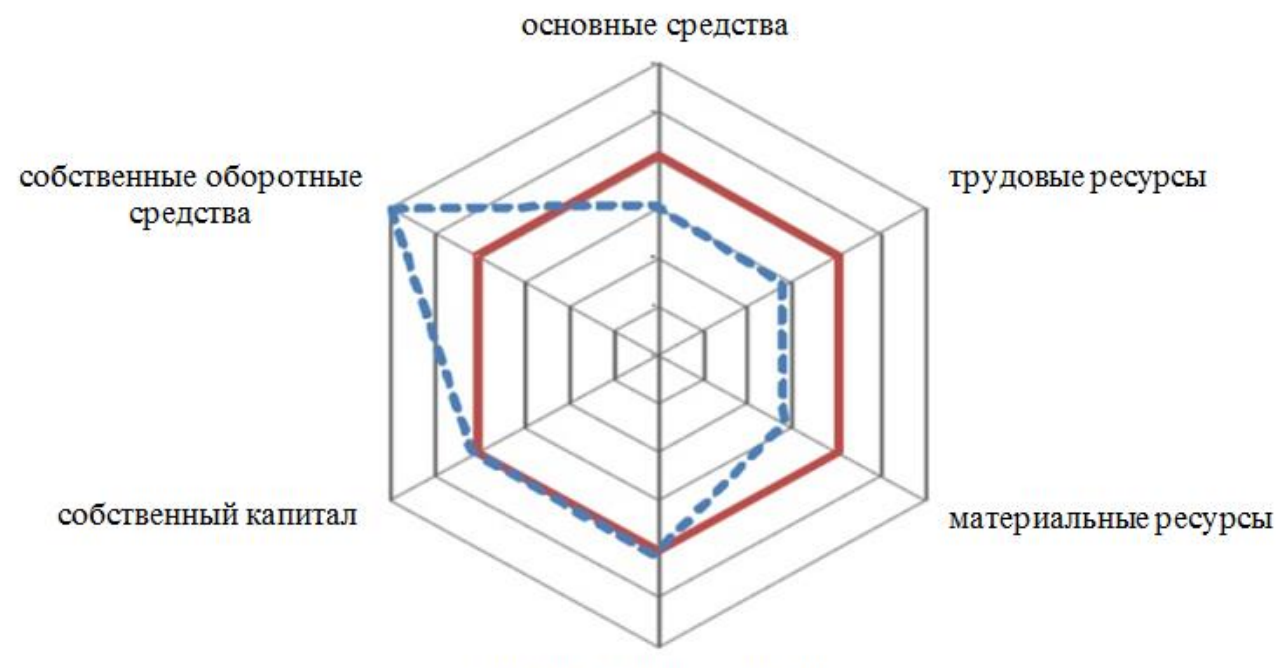

коммерческие операции

-- фактические значения _—нормальные» значения»

\section{Рисунок 1 - Сбалансированность развития ФГУП им. Калинина.}

Проведенный анализ показал, что ФГУП им. Калинина недостаточно эффективно использует ресурсный потенциал, структура капитала соответствует «нормальному» значению, скорость коммерческих операций даже несколько превышает аналогичный среднеотраслевой уровень. При этом в структуре имущества наблюдается необычно высокая для отрасли доля собственных оборотных средств.

Таким образом, нами была выявлена несбалансированность деятельности ФГУП им. Калинина. У организации наблюдаются преимущества в структуре имущества и источников его образования, которые не используются в полной мере вследствие недостаточной эффективности использования имеющихся ресурсов. Руководству организации необходимо сосредоточить усилия на повышении интенсивности использования ресурсной базы, что должно найти свое отражение в увеличении фондоотдачи, производительности труда и материалоотдачи хотя бы до уровня среднеотраслевых значений.

Проведенное исследование показало, что для достижения указанной цели необходимо:

1) сокращение запасов готовой продукции.

В ФГУП им. Калинина наблюдается излишек остатков готовой продукции, что свидетельствует о затоваривании предприятия. Средства, обездвиженные в запасах, не участвуют в обороте и не приносят организации доходов, что ведет к снижению эффективности ее деятельности.

С финансовой точки зрения запасы - это иммобилизованные средства, т.е. средства, в известном смысле отвлеченные из оборота.
Понятно, что без такой вынужденной иммобилизации не обойтись, однако необходимо минимизировать вызываемые этим процессом косвенные потери, с определенной долей условности численно равные доходу, который можно было бы получить, инвестировав соответствующую сумму в какой-то альтернативный проект [3];

2) оптимизация управления денежными потоками.

ФГУП им. Калинина активно наращивает объем денежной наличности на расчетных счетах, которая не участвует в обороте и не приносит организации экономических выгод. При этом организация платежеспособна и абсолютно ликвидна, практически не использует заемные средства и может в любой момент времени погасить все свои обязательства без ущерба для текущей деятельности. В условиях отсутствия «больных» статей в отчетности (убытки, кредиты и займы, не погашенные в срок, просроченная дебиторская и кредиторская задолженность) ситуации, когда потребуется мгновенное погашение обязательств, не может возникнуть, поэтому хранение на расчетных счетах большого объема денежной наличности представляется необоснованным и неэффективным [4].

Свободные и высвободившиеся от продажи излишков готовой продукции денежные средства ФГУП им. Калинина следует направить либо на осуществление финансовых вложений, либо на расширение и модернизацию производства.

Однако ФГУП им. Калинина никогда не осуществляло финансовые вложения и, следовательно, не имеет в этом опыта, к тому же 
в условиях нестабильности экономики страны можно очень быстро потерять свои «вложения». Поэтому более целесообразен второй вариант;

3) обновление основных средств. ФГУП им. Калинина постепенно обновляет материальнотехническую базу производства, но вследствие высокой степени износа оборудования (более 40\%) выбытие основных фондов происходит более быстрыми темпами. Так, только за 2012 г. было списано 25 тракторов, 5 комбайнов и 4 грузовых автомобиля. В то же время за последние два года (2011 и 2012 гг.) было приобретено лишь 8 тракторов, 6 комбайнов и ни одного грузового автомобиля. Очевидно, что данная ситуация не способствует повышению эффективности деятельности организации.

Приобретение высокотехнологичного оборудования позволит сократить затраты труда, времени и материалов на производство продукции, что выразится в сокращении себестоимости производства, росте прибыли хозяйства и возможности осуществлять производство на расширенной основе.

Для обоснования потребности в сельскохозяйственной технике рассмотрим обеспеченность предприятия основными средствами (таблица 1).

Как видно из таблицы площадь пашни в динамике за три года практически не изменялась. А количество тракторов организации в 2012 и 2013 гг. по сравнению с 2011 г. сократилась на 20 единиц. Это привело к увеличению нагрузки на один трактор на 38 га.

Таблица 1

Обеспеченность ФГУП им. Калинина сельскохозяйственной техникой.

\begin{tabular}{|l|c|c|c|c|c|}
\hline \multicolumn{1}{|c|}{ Показатель } & \multirow{2}{*}{2011 г. } & \multirow{2}{*}{2012 г. } & \multirow{2}{*}{2013 г. } & $\begin{array}{c}\text { Отклонение (+,-) в 2013 г. } \\
\text { по сравнено с }\end{array}$ \\
\cline { 5 - 7 } & & & & 2011 г. & 2012 г. \\
\hline Площадь сельскохозяйственных угодий, га & 8917 & 8980 & 8980 & 63 & 0 \\
\hline из них пашня, га & 8917 & 8912 & 8912 & -5 & 0 \\
\hline Площадь посевов зерновых культур, га & 4120 & 3449 & 3615 & -505 & 166 \\
\hline Наличие техники (на конец года), ед.: & & & & & \\
\hline - тракторов & 80 & 60 & 60 & -20 & 0 \\
\hline - комбайнов & 26 & 22 & 22 & -4 & 0 \\
\hline - в т.ч. зерноуборочных & 22 & 18 & 18 & -4 & 0 \\
\hline - сеялки & 14 & 18 & 24 & 10 & 6 \\
\hline - грузовые автомобили & 35 & 31 & 30 & -5 & -1 \\
\hline Нагрузка пашни на один трактор, га & 111 & 149 & 149 & 38 & 0 \\
\hline $\begin{array}{l}\text { Приходится посевов зерновых культур на } \\
\text { один зерноуборочный комбайн, га }\end{array}$ & 187 & 192 & 201 & & \\
\hline
\end{tabular}

Из представленных данных следует, что предприятию необходимо увеличить парк тракторов.

Увеличение площади посевов зерновых в 2013 г. привело к повышению нагрузки на один комбайн на 9 га. При этом следует отметить, что сокращение площади посевов зерновых культур в 2012 г. связано с невысокими результатами реализации данного вида культур в 2011 г. В условиях ожидаемого роста цен на зерно в 2014 г. руководству организации, возможно, следует рассмотреть вопрос о возврате к старой структуре посевов. Это потребует увеличения количества зерноуборочных комбайнов.

Обеспеченность ФГУП им. Калинина сеялками достаточная и наблюдается рост их количества с 14 ед. в 2011 г. до 24 ед. в 2013 г.

Сокращение количества грузовых автомобилей на протяжении трех лет при сохранении грузопотока предприятия негативно отражается на результатах работы хозяйства. Для обеспечения уборки и доставки урожая сельскохозяйственной продукции в агрономические сроки предприятию необходимо увеличить количество данного вида техники.

Проведенные расчеты показали, что организация за счет мобилизации имеющихся резервов без ущерба для текущей деятельности и риска появления неплатежеспособности имеет возможность приобрести 6 тракторов, 3 зерноуборочных комбайна и 4 грузовых автомобиля. По результатам проведенного исследования соблюдение указанных пропорций между видами сельскохозяйственной техники позволит организации получить наибольший эффект. 
Таким образом, в отношении ФГУП им. Калинина можно говорить именно о недостаточной обеспеченности основными средствами, что приводит к невысокой эффективности работы всего предприятия в целом.

Приведенный нами пример показывает, как комплексный подход к анализу эффективности предпринимательской деятельности позволяет оценить сбалансированность развития конкретных субъектов хозяйствования, определить их сильные и слабые стороны, а также разработать стратегию дальнейшего развития.

\section{References:}

1. (2009) Analiz hozjajstvennoj dejatel'nosti: ucheb. posobie / [Barilenko V.I. i dr.]; pod red. V.I. Barilenko. - M.: Izdatel'stvo "Omega-L", 2009. - 414 p.

2. Kravchenko AS (2014) Kompleksnaja ocenka jeffektivnosti predprinimatel'skoj dejatel'nosti sel'skohozjajstvennyh organizacij // Theoretical \& Applied Science. 2014. № 12 (20). pp. 53-56.

3. Kogdenko VG (2011) Jekonomicheskij analiz: uchebnik. - 2-e izd., pererab. i dop. - M.: JuNITI-DANA, 2011. - 399 p.

4. Kovalev VV (2008) Kurs finansovogo menedzhmenta: ucheb. - M.: TK Velbi, Izd-vo Prospekt, 2008. - 448 p.

5. Ljubushin NP (2010) Jekonomicheskij analiz: uchebnik 3-e izd., pererab. i dop. - M.: JuNITIDANA, 2010. - 575 p.

6. Mejer MV (2004) Ocenka jeffektivnosti biznesa / Marshal V. Mejer; [Per. s angl. A.O.
Korsunskij]. - M.: OOO «Vershina», 2004. 272 p.

7. Pereverzev MP, Lunjova AM (2009) Osnovy predprinimatel'stva: uchebnik / Pod obshh. red. prof. M.P. Pereverzeva. - M.: INFRA-M, 2009. $-176 \mathrm{p}$.

8. Savickaja GV (2014) Analiz jeffektivnosti i riskov predprinimatel'skoj dejatel'nosti. Metodologicheskie aspekty / G. V. Savickaja.M.: Infra-M, 2014. - 272 p.

9. Sheremet AD (2006) Kompleksnyj analiz hozjajstvennoj dejatel'nosti. - M.: INFRA-M, 2006. -415 p.

10. Vahrushina MA (2010) Upravlencheskij analiz: ucheb. posobie dlja studentov, obuchajushhihsja po spec. «Buhgalterskij uchet, analiz i audit» / M.A. Vahrushina. - 6-e izd., ispr. - M.: Izdatel'stvo «Omega-L», 2010. - 399 s. 